\title{
Burden of Seasonal Influenza A and B in Panama from 2011 to 2017: An Observational Retrospective Database Study
}

\author{
Juan Miguel Pascale Danilo Franco $\cdot$ Raghavendra Devadiga \\ Rodrigo DeAntonio • Elidia Lourdes Dominguez-Salazar • \\ Gael dos Santos $\cdot$ Patricia Juliao (1)
}

Received: March 29, 2021 / Accepted: July 7, 2021 / Published online: August 23, 2021

(C) The Author(s) 2021

\begin{abstract}
Introduction: Influenza A and B viruses constantly evolve and cause seasonal epidemics and sporadic outbreaks. Therefore, epidemiological surveillance is critical for monitoring their circulation pattern. Trivalent and quadrivalent vaccine formulations are available in Panama (until and since 2016, respectively). Herein, we analysed influenza A and B epidemiological patterns in Panama.

Methods: This was a retrospective descriptive analysis of all laboratory-confirmed influenza
\end{abstract}

Supplementary Information The online version contains supplementary material available at https:// doi.org/10.1007/s40121-021-00501-y.

J. M. Pascale $\cdot$ D. Franco

Instituto Conmemorativo Gorgas de Estudio de

Salud, Panama City, Panama

R. Devadiga

GSK, Bangalore, India

R. DeAntonio

CEVAXIN The Panama Clinic-Sistema Nacional de Investigación, Senacyt, Panama

E. L. Dominguez-Salazar · P. Juliao ( $\square)$

GSK, Panama City, Panama

e-mail: patricia.d.juliao@gsk.com

G. dos Santos

GSK, Wavre, Belgium nasopharyngeal samples recorded between 2011 and 2017 in the nationwide surveillance database of Gorgas Memorial Institute for Health Studies. The analysis involved data relative to demographic information, virus type, subtype and lineage, geographic region, treatment and outcomes. The percentage level of mismatch between circulating and vaccine-recommended B lineage was assessed for each May-October influenza season.

Results: Among 1839 influenza cases, 79.6\% were type A and $20.4 \%$ were type B. Most of them were observed in Panama City (54.7\%) followed by the West (23.2\%) and Central (16.7\%) regions; across all regions, influenza A and B cases were distributed in a $4: 1$ ratio. Overall, approximately half were hospitalized (52.0\% for type A; $45.5 \%$ for type B) and 11 $(0.6 \%)$ died. Treatment, usually antimicrobial, was administered in $15.1 \%$ of cases. Children less than 2 years old were the most affected by this disease. Influenza type A circulated every year, while influenza B only circulated in 2012, 2014 and 2017. In the 2012 May-October influenza B season, the predominant lineage was B/Victoria and a switch to B/Yamagata was observed in 2014. Both lineages co-circulated in 2017 , leading to a $38.9 \%$ B-lineage-level vaccine mismatch.

Conclusion: Influenza A was predominant among all ages and children less than 2 years and inhabitants of Panama City reported the highest circulation rate. In 2017, co-circulation 
of both B lineages led to a vaccine mismatch. Continuous monitoring of seasonal influenza is critical to establish immunization recommendations.

\section{PLAIN LANGUAGE SUMMARY}

Influenza or "flu" is caused by influenza viruses $\mathrm{A}$ and $\mathrm{B}$ and its symptoms range from mild to severe. This virus is constantly evolving; thus, careful monitoring of influenza is important to update immunization and vaccine recommendations yearly. This study used data from surveillance centres in Panama from 2011 to 2017 and evaluated the number of flu cases by age, gender, region, virus type, symptoms, comorbidities, treatment, coinfections with other viruses, and the circulating influenza subtype and the vaccine recommended each year. We found several points: almost $80 \%$ of cases were influenza $\mathrm{A}$; most of the positive samples were found in children less than 2 years old and the Panama city region; more than 50\% of influenza cases needed hospitalization; and in 2017 a mismatch was detected between the circulating influenza subtype and the recommended vaccine. This study helped to better characterize influenza circulation patterns and the burden of the disease during 2011-2017. We concluded that continuous monitoring of the influenza cases is necessary to establish future vaccination recommendations.

Keywords: Influenza A; Influenza B; Influenza vaccine; Panama; Tetravalent flu vaccine; Trivalent flu vaccine; Epidemiology

\section{Key Summary Points}

\section{Why carry out this study?}

Influenza virus (A and B types) constantly evolve causing seasonal epidemics globally.

Analysis and characterization of local data could help better understand the burden and pattern of influenza in Panama.

\section{What was learned from the study?}

A total of 1839 cases of influenza were reported from January 2011 to December $2017 ; 79.6 \%$ were of type A and $20.4 \%$ were of type B. Children less than 2 years old were the most affected $(30.8 \%$ of all cases) and the region of Panama City had the highest circulation rate $(54.7 \%)$. More than half of the cases were hospitalized and a small portion died (0.6\%).

Co-circulation of B/Victoria and $\mathrm{B} /$ Yamagata led to a 38.9\% B-lineage-level trivalent seasonal influenza vaccines (TIVs) mismatch in 2017.

Co-circulation of both B lineages in Panama supports the use of the tetravalent flu vaccine in Panama and neighbouring countries and stresses the importance of continuous monitoring of seasonal influenza for future immunization recommendations.

\section{INTRODUCTION}

Influenza is an acute respiratory infection of varying severity, ranging from asymptomatic to fatal with an annual attack rate estimated at $5-10 \%$ in adults and $20-30 \%$ in children [1], and is responsible for an estimated $290,000-650,000$ respiratory deaths each year [2].

Vaccination is the most effective way to prevent influenza [2]. A recent publication reported that 38 out of the 50 countries and 
territories of Latin America and the Caribbean had an immunization policy for the annual use of seasonal influenza vaccine [3]. Since 2010, trivalent and quadrivalent influenza vaccines (TIVs and QIVs, respectively) have been available each season [4]. Most countries in Latin America use the TIVs (around 90\% in 2017), containing three influenza strains: $\mathrm{A}(\mathrm{H} 1 \mathrm{~N} 1)$, $\mathrm{A}(\mathrm{H} 3 \mathrm{~N} 2)$ and one influenza B lineage (either $\mathrm{B} /$ Victoria or B/Yamagata), mainly because of their lower cost compared to the QIVs (ca. US \$1 per dose versus US \$5 USD per dose, respectively) [3].

The QIVs, which include both subtypes of A and both $\mathrm{B}$ lineages, have been available for use in Panama since 2016 [3, 5]. The Ministry of Health recommends seasonal influenza vaccination for children aged 6 months to 5 years, adults 60 years and older, pregnant women, people with chronic conditions, and specific groups (including healthcare workers, indigenous populations and others) [6]. In 2018, coverage was estimated at $57 \%$ in children and adolescents (from 6 months to 18 years of age) and $100 \%$ in adults aged above 60 years, although these values should be considered carefully [7].

Influenza viruses constantly evolve [8]. During each season, both influenza $\mathrm{A}$ subtypes $\mathrm{H} 1 \mathrm{~N} 1$ and $\mathrm{H} 3 \mathrm{~N} 2$ are present in variable proportions [3]. In addition, two antigenically distinct lineages of influenza type $B$ viruses (B/ Victoria and $\mathrm{B} /$ Yamagata) have been circulating globally for 35 years [9]. The reported effectiveness of influenza vaccines varies substantially with several factors including the type, subtype and lineage of the circulating influenza viruses, the level of match (mismatch) between the vaccine type and the main circulating virus, the time elapsed since vaccination, the region where vaccine effectiveness was measured and case definition specificity (e.g. laboratory-confirmed influenza disease vs. clinical diagnosis of influenza-like illnesses) [8, 10, 11].

Since the 2009 influenza A (H1N1) pandemic, countries of Latin America have strengthened their influenza surveillance systems. Nonetheless, published data on the circulation of influenza B viruses is scarce as identification and reporting of influenza $B$ virus lineages have begun only recently $[3,12,13]$. Information is needed to describe the occurrence and circulation of seasonal influenza $A$ and $B$ viruses, measure the potential mismatch between the B lineage included in the TIVs and the circulating influenza strains and identify clinical features of seasonal influenza $\mathrm{A}$ and $\mathrm{B}$. In this context, the monitoring of seasonal influenza by surveillance platforms is critical to inform vaccine formulation for the next season, assess the impact of seasonal influenza vaccination in any given population, and drive public health policies and decision-making.

Therefore, this study aimed to describe influenza epidemiological patterns, from 2011 to 2017, by geographical region and age, and disease burden in Panama. Both virus A and B and subtypes and lineages were included in the analysis, along with corresponding clinical presentation and outcomes details. Finally, we estimated the percentage of co-circulation of $\mathrm{B}$ lineages, by age and region (West, Central, Panama City and Northeast) and described the mismatch between the $B$ lineage included in the TIVs and the circulating $B$ lineage for the different seasons.

\section{METHODS}

This was a retrospective database study to describe influenza epidemiological patterns and disease burden in Panama. The study included data from all laboratory-confirmed cases of influenza A and/or B reported in the Gorgas Memorial Institute for Health Studies (ICGES) database from January 2011 to December 2017.

\section{Objectives}

The primary objective of this study was to describe influenza A and/or B cases by age group and virus A subtype and B lineage during the period analysed. Additionally, we described clinical features and outcomes, viral coinfections, temporal and geographical distribution, both overall and by subtype and lineage, and we estimated the percentage of co-circulation of influenza B lineages among the study population for the different seasons and describe the 
mismatch with the B lineage included in the TIVs.

\section{Data Source and Patient Selection}

ICGES records the data collected through the national passive surveillance program involving 22 sentinel sites, 10 hospitals and 12 health centres, across the country in accordance with the Pan American Health Organization (PAHO)-Centers for Disease Control and Prevention (CDC) Generic Protocol for Influenza Surveillance (December 2006) [14-16]. By a standard procedure, nasopharyngeal samples from patients with influenza like-illness (ILI) and/or severe acute respiratory infection (SARI) symptoms (supplementary methods) are collected and processed for laboratory confirmation of respiratory infection using multiplex reverse transcription polymerase chain reaction (RT-PCR) and viral isolation and typing of both influenza A and B isolates [17]. Demographic data, date of symptom onset, geographic region, clinical features and outcomes for all influenzapositive subjects were also collected along with sample collection and recorded within the database.

\section{Statistical Analyses}

Demographic characteristics were summarized by group using descriptive statistics and frequency tables were generated for categorical variables. The proportion of individuals with influenza A and/or B and the distribution of influenza cases by month, year and yearly southern hemisphere influenza season (May to October) were tabulated using frequency, percentages, age group, gender, region, signs and symptoms and influenza type. The proportion of individuals with influenza A and/or B was calculated with 95\% confidence intervals (CI) by age and virus subtype/lineage.

The circulating influenza B lineages along with the TIVs, which comprise two influenza A subtypes and one influenza B lineage, were tabulated and evaluated using frequency and percentage for each influenza season. The proportion of mismatched B lineage was calculated as 1 minus the proportion of matched $\mathrm{B}$ lineage and expressed as a percentage.

The statistical analyses were performed using the Statistical Analysis System version 9.3 (SAS Drug Development, SDD).

\section{Compliance with Ethics Guidelines}

The study was performed in accordance with the Declaration of Helsinki, the principles of Good Clinical Practice, and all applicable regulatory requirements, including Good Epidemiological Practice. The study protocol, any subsequent amendments, and other information that required preapproval were reviewed and approved by the Independent Ethics Committee of the ICGES. The ICGES database was fully coded and data were anonymised prior to data processing and analysis so that no third party could link data to an identifiable individual. Therefore, no patient consent was needed.

\section{RESULTS}

From 2011 to 2017 , a total of 15,426 samples were recorded, of which 1839 (11.9\%) were influenza positive (Table S1); 79.6\% (1464 cases) were influenza virus type A and $20.4 \%$ were influenza virus type B (376 cases) (Table 1). The influenza cases were equally distributed between male and female individuals for both influenza A and B (Table 1). More than half of the influenza cases reported were observed in Panama City $(54.7 \%)$ followed by West and Central regions. Influenza $\mathrm{A}$ and $\mathrm{B}$ cases were distributed in a 4:1 ratio across all Panamanian regions (Table 1 ).

Young children less than 2 years old were the most affected by the disease, representing $30.8 \%$ of all influenza-positive samples. In older subjects, influenza cases represent $9.2 \%$ for patients aged 2-4, 15.4\% for patients aged 5-19, $17 \%$ for patients aged $20-37,15.8 \%$ for patients aged $40-59$, and $10.3 \%$ for patients aged 60 years or older. A single case, aged less than 2 years old, was positive for both influenza types $\mathrm{A}$ and $\mathrm{B}$ (Table 1). In all age groups influenza type $A$ was more frequent. In older adults (60 years or older), influenza burden was almost 
Table 1 Demographic characteristics by influenza type

\begin{tabular}{llll}
\hline & $\begin{array}{l}\text { Influenza A } \\
\boldsymbol{N}=\mathbf{1 4 6 4}\end{array}$ & $\begin{array}{l}\text { Influenza B } \\
\boldsymbol{N}=\mathbf{3 7 6}\end{array}$ & $\begin{array}{l}\text { Influenza A and/or B } \\
\boldsymbol{N}=\mathbf{1 8 3 9}\end{array}$ \\
\hline Age group, years, $n(\%)^{\mathrm{a}}$ & & & \\
$<2$ & $448(30.6)$ & $120(31.9)$ & $567(30.8)^{\mathrm{b}}$ \\
$2-4$ & $123(8.4)$ & $47(12.5)$ & $170(9.2)$ \\
$5-19$ & $170(11.6)$ & $113(30.1)$ & $283(15.4)$ \\
$20-39$ & $274(18.7)$ & $39(10.4)$ & $313(17.0)$ \\
$40-59$ & $254(17.3)$ & $37(9.8)$ & $291(15.8)$ \\
$\geq 60$ & $180(12.3)$ & $10(2.7)$ & $190(10.3)$ \\
Unspecified & $15(1.0)$ & $10(2.7)$ & $25(1.4)$ \\
Gender, $n(\%)^{\mathrm{a}}$ & & $193(51.3)$ & $905(49.2)$ \\
Male & $712(48.6)$ & $183(48.7)$ & $909(49.4)^{\mathrm{b}}$ \\
Female & $727(49.7)$ & $0(0.0)$ & $25(1.4)$ \\
Unspecified & $25(1.7)$ & & \\
Region, $n(\%)^{\mathrm{a}}$ & & $82(21.8)$ & $327(23.2)$ \\
West & $345(23.6)$ & $64(17.0)$ & $308(16.7)$ \\
Central & $244(16.7)$ & $201(53.5)$ & $1006(54.7)$ \\
Panama City & $805(55.0)$ & $12(3.2)$ & $59(3.2)^{\mathrm{b}}$ \\
Northeast & $48(3.3)$ & $17(4.5)$ & $39.1)$ \\
Unspecified & $22(1.5)$ & &
\end{tabular}

Region: West: Bocas del Toro, Chiriquí and Comarca Ngäbe-Buglé; Central: Coclé, Herrera, Los Santos and Veraguas; Panama City: Panamá Este, Panamá Oeste, Panamá Metro and San Miguelito; Northeast: Colón, Darien and Guna Yala a $\%=(n / N) \times 100, N=$ Number of influenza-positive samples, and $n=$ number of positive specimens in a given category

${ }^{\mathrm{b}}$ One specimen sample was positive for both influenza A and B

exclusively associated with type A while type B was mostly present in younger people (below 20 years of age) (Fig. 1 and Table S2).

\section{Influenza Cases by Virus Subtype and Lineage}

Influenza A circulated every year, with a peak in cases between May and October (Fig. 2a). The highest number was recorded in 2016 and the predominant subtype in circulation was $\mathrm{A}(\mathrm{H} 1 \mathrm{~N} 1)$ (815 cases, $98.5 \%$ of A cases) (Fig. 2a and Table S1). Moreover, among all the influenza cases, the subtype influenza A (H1N1) was predominant in circulation (Fig. 1), with more than $40 \%$ of influenza-positive individuals in all the regions (Table S3).

Influenza B circulated in five of seven years (Fig. 2b, Table S1), principally during 2012 (64.7\%, 178 of 275 cases), 2014 (39.3\%, 55 of 140 cases) and 2017 (52.7\%, 137 of 260 cases). B/Victoria was the predominant lineage in 2012 (100.0\%, 178 of 178 B cases), B/Yamagata in 2014 (100.0\%, 55 of 55 B cases), while both lineages coexisted in 2017 (B/Victoria: 32.7\%, 85 of $137 \mathrm{~B}$ cases; B/Yamagata: $20.0 \%, 52$ of 137 cases) (Fig. 2b, Table S1). B/Yamagata lineage was the 
600

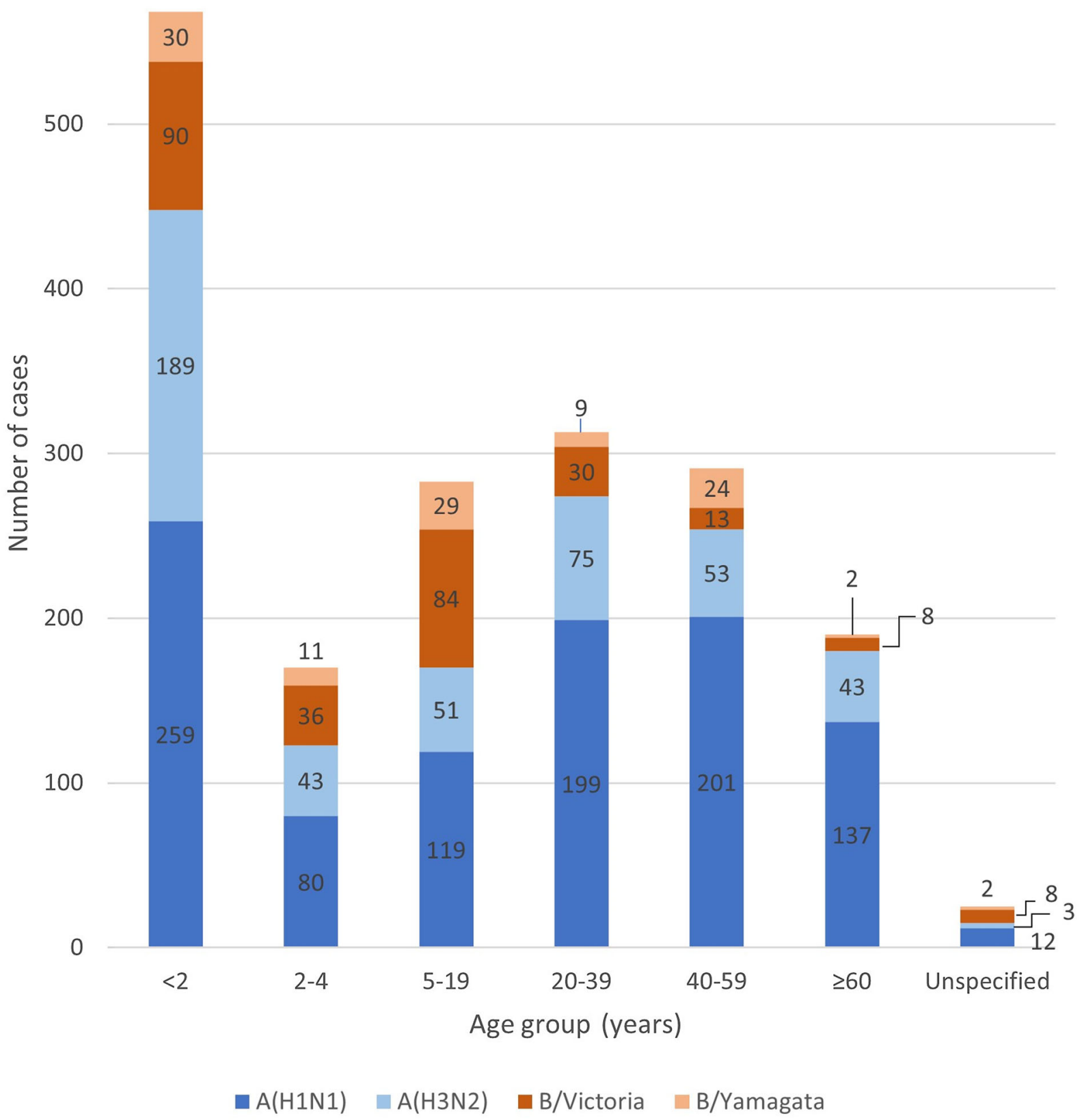

Fig. 1 Distribution of influenza cases by A subtype or B lineage and by age, between January 2011 and December 2017

less widespread among all the influenza subtypes/lineage with less than $7 \%$ of cases in all regions except in the Northeast where the B/Victoria lineage was the less frequent (Table S3).

\section{Occurrence of Clinical Symptoms and Outcomes}

As shown in Table 2, the most commonly reported symptoms from 2011 to 2017 were cough, fever, and rhinorrhoea, that appeared 


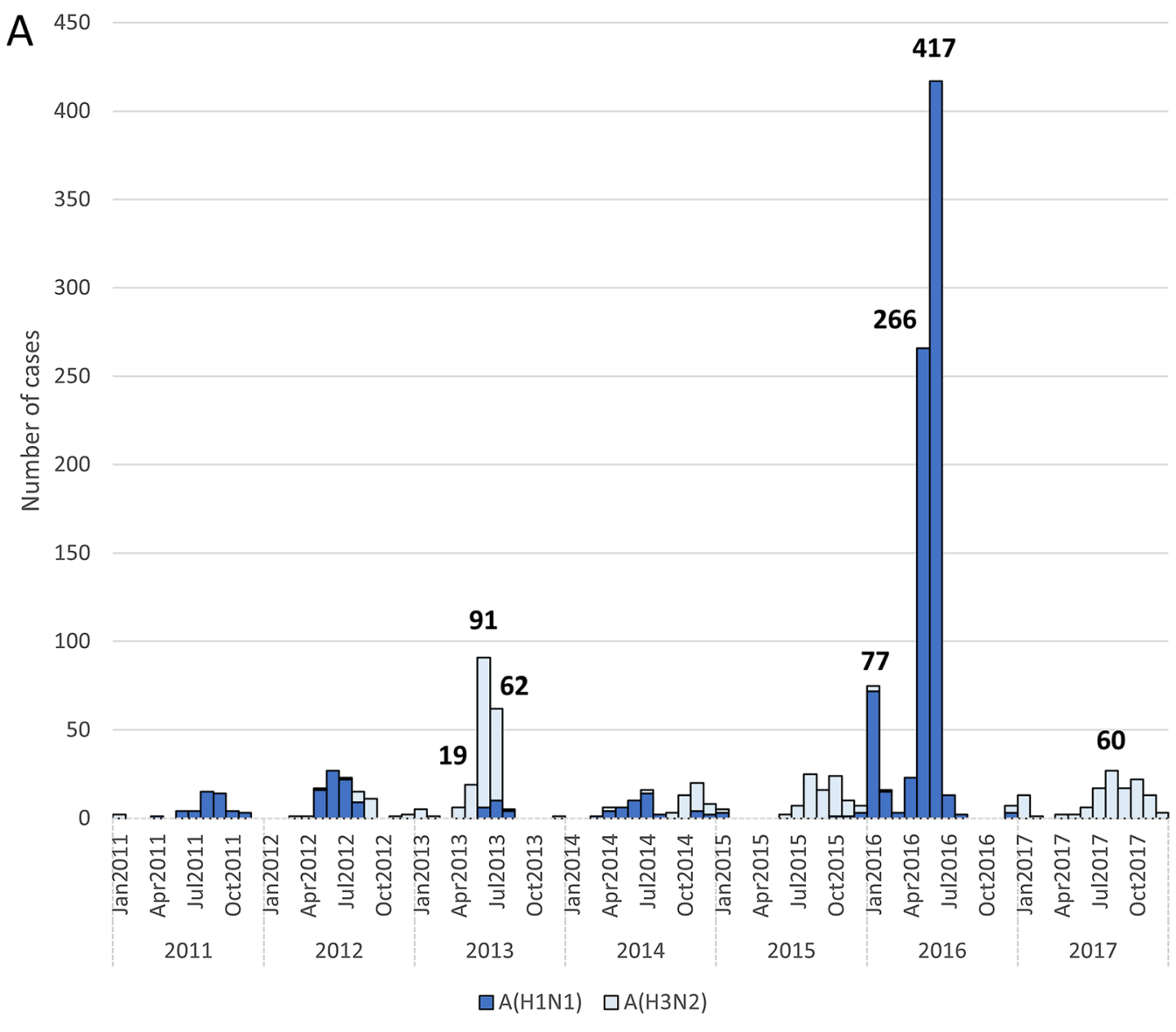

B 150

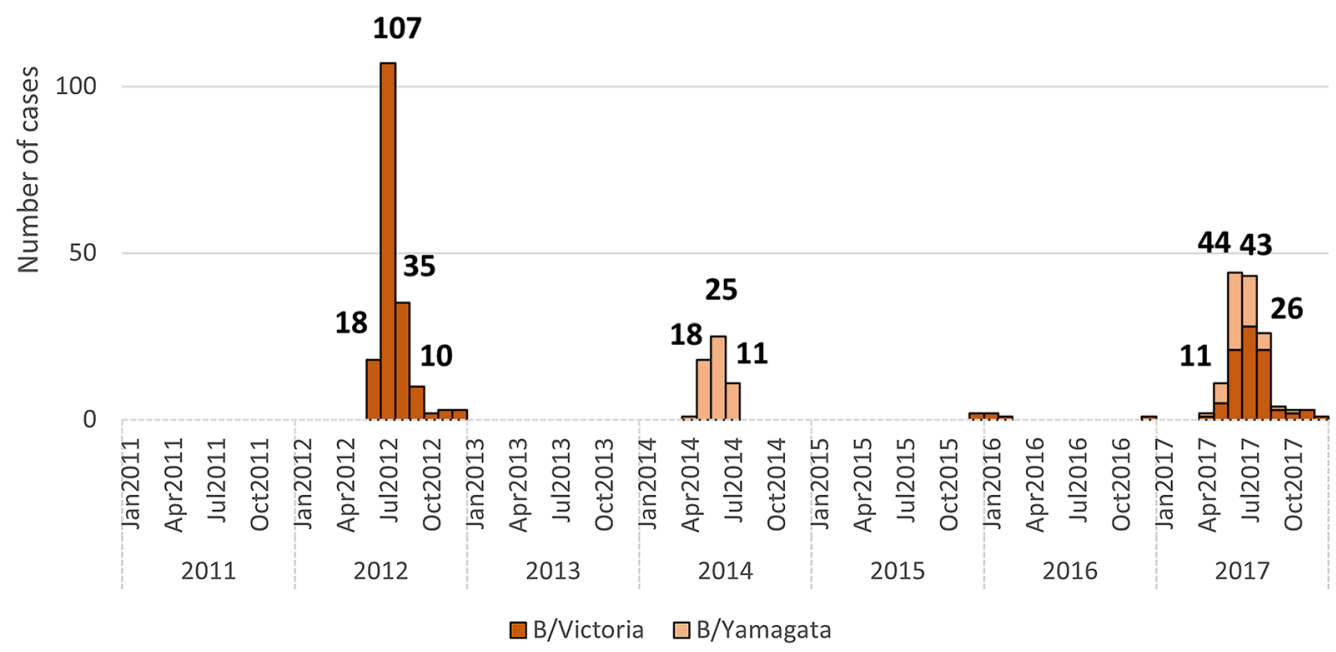

Fig. 2 Monthly distribution of influenza-positive samples and A subtype/B lineage from January 2011 to December 2017. a Influenza A by subtypes. b Influenza B by lineage 
uniform across all influenza types and the most frequent co-morbidities were hypertension, asthma and diabetes (Table 2). Treatment, most commonly antimicrobials, was administered in $15.1 \%$ of all influenza cases (Table 2). Half of the cases recorded were inpatients (hospitalized), followed by outpatients and 11 patients who died (Table 2). The fatality rate increased after 2015 when the first deaths were recorded (10 in 2016 and 1 in 2017) (Fig. 3). When examining outcomes by age group, the highest proportion of death reported was in older adults (at least 60 years old) $(3 / 190,1.6 \%)$, followed by less than 2 years old $(4 / 567,0.7 \%), 5-19$ years old $(2 / 283,0.7 \%)$ and $40-59$ years old $(2 / 291$, $0.7 \%)$. No deaths were reported in those aged 2-4 years and 20-39 years. Patients aged less than 2 years had the highest hospitalizations $(443 / 567,78.1 \%)$ followed by older adults (at least 60 years old) $(111 / 190,58.4 \%)$ and then patients aged $2-4$ years $(95 / 170,55.9 \%)$, 40-59 years $(109 / 291,37.5 \%), 5-19$ years $(101 / 283$, $35.7 \%$ ) and $20-39$ years (70/313, 22.4\%). By region, the proportion of deaths was less than $2 \%$ [Central $(2 / 308,0.6 \%)$, Northeast $(1 / 59$, $1.7 \%)$, Panama (5/1006, 0.5\%), West (3/427, $0.7 \%)]$ while hospitalization was highest in the Northeast $(51 / 59,86.4 \%)$ followed by Central $(255 / 308,82.8 \%)$, West $(275 / 427,64.4 \%)$ and Panama (345/1006, 34.3\%).

As reported in Table 2, 130 individuals were positive for other pathogens. The viral coinfections most frequently reported were human rhinovirus (HRV) followed by parainfluenza virus type 3 (PIV-3) and respiratory syncytial virus (RSV), and adenovirus (ADV).

\section{Circulating Influenza B and Vaccine Lineage Recommendation Included in TIVs}

There were 1580 overall identified cases of influenza during the southern hemisphere influenza seasons (May-October) from 2011 to 2017 (Table S4). In the May-October influenza seasons 2012 and 2014 only one B lineage (B/ Victoria and B/Yamagata, respectively) was in circulation and matched the TIVs recommendation for those corresponding years. However, in the May-October season 2017, a mismatch between the recommended TIVs containing the $\mathrm{B} /$ Victoria lineage and circulating influenza B lineages (B/Yamagata in $38.9 \%$ of the cases) was observed (Table S5).

\section{DISCUSSION}

This descriptive study represents a retrospective analysis of all the laboratory-confirmed influenza A and B cases in the ICGES database from January 2011 to December 2017 in Panama. This national surveillance program has been implemented in Panama since 2010, and the ICGES laboratory, a reference laboratory in Panama and Central America, plays an integral role in it. Influenza occurred following a southern hemisphere seasonal pattern with both types A and B circulating within the population and peaking between May and October. We found that influenza type A was predominant in all age groups and years; its highest number of cases was recorded in 2016 and involved the subtype A(H1N1). The Panama City region had the highest number of influenza cases and overall children aged less than 2 years old were the most affected by this disease. Also, approximately half of the reported influenza cases required hospitalization with a marked increase from 2015 to 2017. Co-circulation of both influenza B lineages was only observed in 2017, in $38.9 \%$ of the cases, and caused a mismatch between the $B$ lineages and the recommended TIV for that year.

In Panama, seasonal influenza vaccination campaigns start between April and May, i.e. 4-6 weeks before the beginning of the rainy season and associated increase in respiratory diseases [18]. In terms of influenza circulation, over $80 \%$ of influenza-positive cases were reported from May to October of each year, which corresponds to the usual defined period for southern hemisphere influenza season [19]. About half of the positive samples analysed in this study were collected in 2016, most likely because of a virulent outbreak of seasonal influenza. That year, the predominant subtype/lineage in circulation was $\mathrm{A}(\mathrm{H} 1 \mathrm{~N} 1)$ with $99 \%$ of the total cases. Influenza B spread 
Table 2 Occurrence of clinical symptoms and outcomes

\begin{tabular}{|c|c|c|c|}
\hline & $\begin{array}{l}\text { Influenza A } \\
N=1464\end{array}$ & $\begin{array}{l}\text { Influenza B } \\
N=376\end{array}$ & $\begin{array}{l}\text { Influenza } A \text { and/or } B \\
N=1839\end{array}$ \\
\hline \multicolumn{4}{|c|}{ Signs and symptoms, $n(\%)^{\mathrm{a}}$} \\
\hline Cough & $1288(88.0)$ & $312(83.0)$ & $1599(86.9)$ \\
\hline Fever & $1261(86.1)$ & $325(86.4)$ & $1585(86.2)$ \\
\hline Rhinorrhoea & $989(67.6)$ & $284(75.5)$ & $1272(69.2)$ \\
\hline Sore throat & $610(41.7)$ & $145(38.6)$ & $754(41.0)$ \\
\hline Dyspnoea & $548(37.5)$ & $82(21.8)$ & $629(34.2)$ \\
\hline Headache & $85(5.8)$ & $32(8.5)$ & $117(6.4)$ \\
\hline Weakness & $110(7.5)$ & $6(1.6)$ & $116(6.3)$ \\
\hline Others $^{b}$ & $183(12.5)$ & $24(6.4)$ & $207(11.6)$ \\
\hline \multicolumn{4}{|l|}{ Comorbidities, $n(\%)^{\mathrm{a}}$} \\
\hline Hypertension & $73(5.0)$ & $6(1.6)$ & $79(4.3)$ \\
\hline Asthma & $45(3.1)$ & $6(1.6)$ & $51(2.8)$ \\
\hline Diabetes & $41(2.8)$ & $0(0.0)$ & $41(2.2)$ \\
\hline Pregnancy & $24(1.6)$ & $3(0.8)$ & $27(1.5)$ \\
\hline Obesity & $20(1.4)$ & $1(0.3)$ & $21(11.0)$ \\
\hline Immunosuppressed & $20(1.4)$ & $3(0.8)$ & $23(1.3)$ \\
\hline Others $^{c}$ & $35(2.4)$ & $4(1.1)$ & $39(2.1)$ \\
\hline \multicolumn{4}{|l|}{ Treatment, $n(\%)^{\mathrm{a}}$} \\
\hline Treatment & $237(16.2)$ & $41(10.9)$ & $278(15.1)$ \\
\hline Antiviral & $51(3.5)$ & $8(2.1)$ & $59(3.2)$ \\
\hline Antimicrobial & $176(12.0)$ & $36(9.6)$ & $212(11.5)$ \\
\hline \multicolumn{4}{|c|}{ Clinical outcome $n(\%)^{\mathrm{a}}$} \\
\hline Outpatients & $648(44.3)$ & $158(42.0)$ & $806(43.8)$ \\
\hline Hospitalised & $761(52.0)$ & $171(45.5)$ & $931(50.6)$ \\
\hline Dead & $11(0.8)$ & $0(0.0)$ & $11(0.6)$ \\
\hline Unspecified & $44(3.0)$ & $47(12.5)$ & $91(4.9)$ \\
\hline \multicolumn{4}{|l|}{ Coinfections $n(\%)^{\mathrm{a}}$} \\
\hline Total & $102(7.0)$ & $29(7.7)$ & $130(7.1)$ \\
\hline ADV & $15(14.7)$ & $2(6.9)$ & $17(13.1)$ \\
\hline HRV & $35(34.3)$ & $7(24.1)$ & $42(32.3)$ \\
\hline PIV-3 & $22(21.6)$ & $3(10.3)$ & $25(19.2)$ \\
\hline RSV & $14(13.7)$ & $11(37.9)$ & $25(19.2)$ \\
\hline
\end{tabular}


Table 2 continued

\begin{tabular}{llll}
\hline & $\begin{array}{l}\text { Influenza A } \\
\boldsymbol{N}=\mathbf{1 4 6 4}\end{array}$ & $\begin{array}{l}\text { Influenza B } \\
\boldsymbol{N}=\mathbf{3 7 6}\end{array}$ & $\begin{array}{l}\text { Influenza A and/or B } \\
\boldsymbol{N}=\mathbf{1 8 3 9}\end{array}$ \\
\hline Other coinfections $^{\mathrm{d}}$ & $16(15.7)$ & $6(20.7)$ & $22(16.7)$ \\
\hline
\end{tabular}

$A D V$ adenovirus, $H R V$ human rhinovirus, $P I V-3$ parainfluenza virus type $3, R S V$ respiratory syncytial virus, $H M P V$ Metapneumovirus, $P I V-1$ parainfluenza virus type 1, PIV-2 parainfluenza virus type 2, HIV/AIDS human immunodeficiency virus/ acquired immunodeficiency syndrome

a $\%=(n / N) \times 100, N=$ Number of influenza positive samples, and $n=$ number of positive specimens in a given category

b Included all cases with a frequency $<3 \%$ in influenza A and/or B: vomiting (40 cases, 2.2\%); diarrhea (43 cases 2.3\%); myalgias ( 43 cases, $2.3 \%)$; nausea ( 9 cases, $0.5 \%)$; wheezing ( 3 cases, $0.2 \%)$; apnoea $(2$ cases, $0.1 \%)$; tachypnoea $(2$ cases, $0.1 \%)$; arthralgia (11 cases, $0.6 \%)$; chest pain (6 cases, $0.3 \%)$; convulsion (8 cases, $0.4 \%)$; hyporexia (16 cases, $0.9 \%)$; irritability ( 7 cases, $0.4 \%)$; odynophagia ( 1 case, $0.1 \%)$; abdominal pain ( 2 cases, $0.1 \%)$; conjunctivitis ( 3 cases, $0.2 \%)$; cyanosis $(1$ case, $0.1 \%)$ and chills ( 10 cases, $0.5 \%)$

${ }^{c}$ Included all cases with frequency $<1$ : tobacco use ( 7 cases, $\left.0.4 \%\right)$; alcoholism ( 2 cases, $\left.0.1 \%\right)$; cardiovascular disease (11 cases, $0.6 \%)$; chronic obstructive pulmonary disease ( 8 cases, $0.4 \%)$; chronic renal insufficiency ( 8 cases, $0.4 \%)$; HIV/AIDS (3 cases, $0.2 \%)$

${ }^{\mathrm{d}}$ Included all coinfections with frequency < 10\%: ADV \& HRV ( 1 case, $0.8 \%$ ); HMPV (10 cases, 7.7\%); HMPV \& PIV-3 ( 1 case, $0.8 \%$ ); influenza ( 1 case, $0.8 \%$ ); PIV-1 \& ADV ( 1 case, $0.8 \%)$; PIV-1 \& PIV-3 (1 case, $0.8 \%) ;$ PIV-2 (2 cases, $1.5 \%$ ); PIV-3 \& HRV (2 cases, $1.5 \%) ;$ RSV \& PIV-3 (1 case, $0.8 \%)$; RSV \& HRV (1 case, $0.8 \%)$

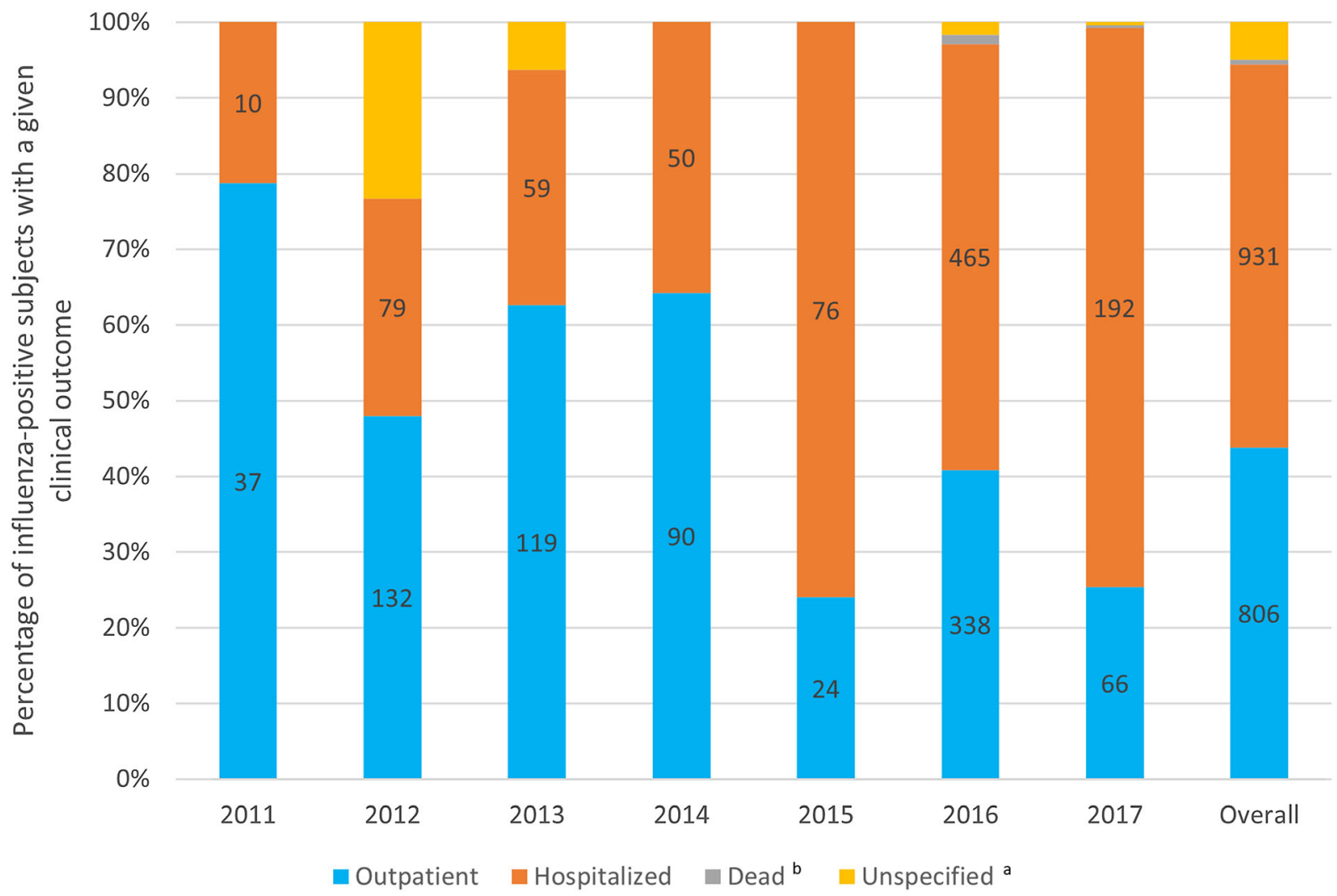

Fig. 3 Frequency of clinical outcomes of influenza cases. ${ }^{a}$ Number of unspecified patients in the figure: 64 in 2012; 12 in 2013; 14 in 2016; 1 in 2017; 91 overall. bumber of dead patients in the figure: 10 in 2016; 1 in 2017; 11 overall 
mainly during three of the seven influenza seasons included in our analyses (2012, 2014 and 2017) and was responsible for almost a fourth of all seasonal cases.

This study showed that influenza A and B virus affected male and female individuals in equal proportion. Among all age groups influenza $\mathrm{A}$ was the most frequent virus type, involving nearly $80 \%$ of the total cases in circulation. Our results are in line with previously published findings, where a marked disease burden was found in subjects less than 2 years of age [20]. Low influenza vaccination coverage has been reported previously within this age group (53\% in 2018); thus, future analysis exploring vaccination coverage and on vaccine effectiveness within this context would be of interest [7].

Nearly $75 \%$ of all influenza B cases were reported among young people (aged less than 20 years old) with young children less than 2 years old representing $31.9 \%$, children $2-$ 4 years old representing $12.5 \%$ and children 5-19 years old representing $30.1 \%$. In these age groups B/Victoria virus infections were predominant over B/Yamagata. By contrast, only $5 \%$ of influenza cases reported in older adults were due to type $B$ virus. The higher burden of influenza B in young children and adolescents has been reported earlier [21-23]. Furthermore, previous studies have indicated an increased vulnerability among younger people to B/Victoria virus infections compared with older age groups, while older people were at increased risk of infection by B/Yamagata [9, 24-26].

According to the present data, the highest number of cases of influenza A and/or B during the study period was reported in Panama City. The increased number of influenza cases observed in this region as compared to the other regions of Panama could be due to a better accessibility to healthcare centres or clinics by the individuals living in the capital, to higher population density and better surveillance in this region [14].

More than half of the overall reported influenza cases required hospitalization and a small portion of influenza patients died (0.6\%). In 2014 , the mortality per 100 inhabitants was lower in Panama $(0.58 \%)$ than in the rest of
Central America: Belize 0.75\%, El Salvador $0.73 \%$, Honduras $0.69 \%$ and Nicaragua $0.65 \%$, with only one exception, Costa Rica, with a rate of $0.44 \%$ [14]. This difference might be explained by the number of hospital beds per 1000 inhabitants, which in Panama ( 2.3 beds) is highly superior to the average in Central America (1.16 beds) [14]. After 2014, both the absolute number and proportion of hospitalizations and deaths among influenza cases rose, indicating an increased severity of the disease linked to the subtype/lineage of circulating viruses at that time (driven mainly by the outbreak reported in 2016), an improvement in the surveillance system or a combination of both. In 2017, after implementation of the QIVs vaccines in Panama, the mortality was even lower compared with the other Central America countries (Panama 0.46\%): Belize 0.60\%, El Salvador $0.70 \%$, Honduras $0.50 \%$, Nicaragua $0.50 \%$ and Costa Rica 0.5\% [27]. Viral coinfections were reported as well during this study with $7.3 \%$ of influenza cases positive for other pathogens. As coinfections may lead to increased severity [28], it is important to characterize in this population and essential in clinical management as well as future diagnostic procedures.

During the 2017 influenza season, the cocirculation of both $\mathrm{B} /$ Victoria and $\mathrm{B} /$ Yamagata lineages in Panama was detected. This fact provoked a mismatch between the recommended TIVs (B/Victoria in 2017) in 38.9\% of the cases. Similar cases were reported that year in other countries. A large study encompassing 19 countries of Latin America and the Caribbean revealed a vaccine mismatch between the predominant $\mathrm{B}$ lineage and that included in the TIVs in one third of the 63 influenza seasons analysed [3]. Another study detected a co-circulation of B/Victoria and B/Yamagata in a third of influenza seasons in Brazil [29]. In addition, recently, an Italian study found a continuous co-circulation of both B lineages between 2004 and 2017 and calculated a mismatch between the circulating B lineage and the TIV B lineage in about two-thirds of cases [9].

As a result of changes in the annual circulation of influenza B, QIVs with both influenza $\mathrm{B}$ lineages are recommended. In case of 
mismatch between the circulating and TIV lineages or co-circulation of different influenza B lineages, as reported in the 2017 influenza season, there is a significant risk of experiencing suboptimal protection from the TIVs as a result of the missing $\mathrm{B}$ lineage. This risk could be mitigated by using QIVs. Further studies of influenza B burden, potential TIV cross protection and QIV cost-effectiveness in the region of Latin America and the Caribbean are needed $[3,30]$.

This study has several limitations. The study was based on a database linked to a national passive sentinel surveillance system for respiratory diseases, which had limitations associated with underreporting, unavailability or incompleteness of data (no defined denominator) [31]. Passive surveillance systems might exclude people who are outside the catchment area of the sentinel sites or with mild disease that do not visit the healthcare sites. This study did not explore the vaccination status of the reported influenza cases nor the vaccination coverage of the Panamanian population and therefore cannot contribute to informing about vaccine effectiveness in Panama. However, within this study most cases and hospitalizations occurred in children where published vaccination coverage appeared low and may be an area of exploration in future studies. Table 2 does not clarify these points.

Also, since we noticed a higher proportion of influenza type B virus over type A in some age groups, further analyses assessing the association between hospitalization and patient age need to be done; this may provide valuable information about the groups to target with influenza vaccination recommendation. Finally, the identification of the influenza viruses associated with hospitalizations would also contribute to the characterization of the burden due to specific influenza types, A subtypes and B lineages.

\section{CONCLUSION}

The present analysis of 2011-2017 national surveillance data revealed influenza type A as the predominant type over the study period and among all ages, with children less than 2 years old being the most affected and the region of Panama City having the highest circulation rate. More than $50 \%$ of the cases were hospitalized and a small portion died (0.6\%). Most cases were detected in 2016, with an outbreak of the $A(H 1 N 1)$ subtype. In 2017, a co-circulation of both $\mathrm{B} /$ Victoria and $\mathrm{B} /$ Yamagata led to a mismatch between TIVs and the circulating viruses. As a result of virus co-circulation and difficulties to predict the circulating influenza subtypes and lineages in the next season, continuous monitoring of influenza and the use of QIVs remain important to reduce the burden of influenza disease.

\section{Acknowledgements}

Funding GlaxoSmithKline Biologicals SA funded this study (205049) and covered all costs associated with the development and publication of this manuscript including the journal's rapid service fee.

Authorship All named authors meet the International Committee of Medical Journal Editors (ICMJE) criteria for authorship for this article, take responsibility for the integrity of the work as a whole, and have given their approval for this version to be published.

Authors Contribution Juan Miguel Pascale, Danilo Franco, Raghavendra Devadiga, Rodrigo DeAntonio, Elidia Lourdes DominguezSalazar, Gael dos Santos and Patricia Juliao participated to the concept, design, analysis, draft and review of this manuscript.

Medical Writing, Editorial, and Other Assistance The authors would like to thank Business \& Decision Life Sciences platform for editorial assistance and manuscript coordination, on behalf of GSK. Pierre-Paul Prévot coordinated manuscript development and editorial support. Marie Cloes provided medical writing support.

Disclosures Raghavendra Devadiga, Elidia Lourdes Dominguez-Salazar, Gael dos Santos and Patricia Juliao are employed by the GSK group of companies. Gael dos Santos, Elidia Lourdes Dominguez-Salazar and Patricia Juliao 
hold shares in the GSK group of companies. Rodrigo DeAntonio was an employee of the GSK group at the time of this study. Juan Miguel Pascale and Danilo Franco declare no financial relationships and activities, and all authors declare no non-financial relationships and activities.

Compliance with Ethics Guidelines The study was performed in accordance with the Declaration of Helsinki, the principles of Good Clinical Practice, and all applicable regulatory requirements, including Good Epidemiological Practice. The study protocol, any subsequent amendments, and other information that required preapproval were reviewed and approved by the Independent Ethics Committee of the ICGES. The ICGES database was fully coded and data were anonymised prior to data processing and analysis so that no third party could link data to an identifiable individual. Therefore, no patient consent was needed.

Data Availability The datasets generated and/ or analysed during the current study are availablefrom the corresponding author on reasonable request.

Prior Presentation Some of the study results included in this manuscript were presented at the $18^{\text {th }}$ International Congress on Infectious Diseases, March 1-4, 2018, Buenos Aires, Argentina.

Open Access This article is licensed under a Creative Commons Attribution-NonCommercial 4.0 International License, which permits any non-commercial use, sharing, adaptation, distribution and reproduction in any medium or format, as long as you give appropriate credit to the original author(s) and the source, provide a link to the Creative Commons licence, and indicate if changes were made. The images or other third party material in this article are included in the article's Creative Commons licence, unless indicated otherwise in a credit line to the material. If material is not included in the article's Creative Commons licence and your intended use is not permitted by statutory regulation or exceeds the permitted use, you will need to obtain permission directly from the copyright holder. To view a copy of this licence, visit http:// creativecommons.org/licenses/by-nc/4.0/.

\section{REFERENCES}

1. World Health Organization. Seasonal influenza. https://www.who.int/ith/diseases/influenza_ seasonal/en/. Accessed 12 May 2020.

2. World Health Organization. Influenza (seasonal) fact sheet. https://www.who.int/news-room/factsheets/detail/influenza-(seasonal) Accessed 13 May 2020 .

3. Palekar A, Rodriguez A, Avila C, et al. Patterns of influenza B circulation in Latin America and the Caribbean, 2010-2017. PLoS One. 2019;14: e0219595.

4. Hannoun C. The evolving history of influenza viruses and influenza vaccines. Expert Rev Vaccines. 2013;12:1085-94.

5. Jamotte A, Clay E, Macabeo B, et al. Public health impact and economic benefits of quadrivalent influenza vaccine in Latin America. Hum Vaccin Immunother. 2017;13:877-88.

6. Comisión Nacional Asesora de Prácticas de Inmunización. Esquema Nacional de Vacunación - Revisado 16 de noviembre 2016. In: Ministerio de Salud - Republica de Panama, ed, 2017.

7. Pan American Health Organization, World Health Organization, Regional Office for the Americas. Immunization in the Americas - 2019 Summary. https://www.paho.org/en/documents/

immunization-americas-2019-summary. Accessed 20 Jun 2020.

8. Dos Santos G, Neumeier E, Bekkat-Berkani R. Influenza: can we cope better with the unpredictable? Hum Vaccin Immunother. 2016;12: 699-708.

9. Puzelli S, Di Martino A, Facchini M, et al. Co-circulation of the two influenza B lineages during 13 consecutive influenza surveillance seasons in Italy, 2004-2017. BMC Infect Dis. 2019;19:990.

10. Belongia EA, Simpson MD, King JP, et al. Variable influenza vaccine effectiveness by subtype: a systematic review and meta-analysis of test-negative design studies. Lancet Infect Dis. 2016;16:942-51.

11. Young B, Sadarangani S, Jiang L, Wilder-Smith A, Chen MI. Duration of influenza vaccine 
effectiveness: a systematic review, meta-analysis, and meta-regression of test-negative design casecontrol studies. J Infect Dis. 2018;217:731-41.

12. El Omeiri N, Azziz-Baumgartner E, Thompson MG, et al. Seasonal influenza vaccine effectiveness against laboratory-confirmed influenza hospitalizations-Latin America, 2013. Vaccine. 2018;36: 3555-66.

13. Saavedra G, Guerra I, Cisneros J, Quiroz E. Influenza en Panama. 1999-2005. http://www.gorgas.gob.pa/ BiblioGorgas/pdf/Influenza\%20en\%20panama\% 201999-2005.\%20Gloriela\%20Saavedra.pdf. Accessed 20 Dec 2020.

14. Fischer J, Cerpa M, Mendez J, et al. Influenza and other respiratory virus surveillance systems in the Americas 2014. https://www.paho.org/hq/ dmdocuments/2014/2014-cha-influenza-orvsurveillance-americas.pdf. Accessed 2 Dec 2020.

15. Nieto-Guevara J, Sosa N, García M, Martinez A, Castillo M. 2009 Influenza A (H1N1) in Panama: a disease affecting children with a benign course. J Infect Dev Ctries. 2011;5:664-8.

16. PAHO-CDC. Generic protocol for influenza surveillance (2006). https://www.paho.org/english/ ad/dpc/cd/flu-snl-gpis.pdf. Accessed 2 Dec 2020.

17. WHO. CDC protocol of realtime RT-PCR for influenza A (H1N1). http://www.who.int/csr/ resources/publications/swineflu/realtimeptpcr/en/ index.htm. Accessed 2 Dec 2020.

18. Hirve S, Newman LP, Paget J, et al. Influenza seasonality in the tropics and subtropics - when to vaccinate? PLoS One. 2016;11:e0153003.

19. Durand LO, Cheng PY, Palekar R, et al. Timing of influenza epidemics and vaccines in the American tropics, 2002-2008, 2011-2014. Influenza Other Respir Viruses. 2016;10:170-5.

20. Sullivan SG, Price OH, Regan AK. Burden, effectiveness and safety of influenza vaccines in elderly, paediatric and pregnant populations. Ther Adv Vaccines Immunother. 2019;7:2515135519826481.

21. Caini S, Huang QS, Ciblak MA, et al. Epidemiological and virological characteristics of influenza B: results of the Global Influenza B Study. Influenza Other Respir Viruses. 2015;9(Suppl 1):3-12.

22. Moa AM, Muscatello DJ, Turner RM, MacIntyre CR. Epidemiology of influenza B in Australia:
2001-2014 influenza seasons. Influenza Other Respir Viruses. 2017;11:102-9.

23. Beaute J, Zucs P, Korsun N, et al. Age-specific differences in influenza virus type and subtype distribution in the 2012/2013 season in 12 European countries. Epidemiol Infect. 2015;143:2950-8.

24. Vijaykrishna D, Holmes EC, Joseph U, et al. The contrasting phylodynamics of human influenza B viruses. Elife. 2015;4:e05055.

25. Socan M, Prosenc K, Ucakar V, Berginc N. A comparison of the demographic and clinical characteristics of laboratory-confirmed influenza B Yamagata and Victoria lineage infection. J Clin Virol. 2014;61: 156-60.

26. Sharabi S, Drori Y, Micheli M, et al. Epidemiological and virological characterization of influenza B virus infections. PLoS One. 2016;11:e0161195.

27. Sosa P, Couto P, Rodriguez A, Charles M, Leite J, Palekar R. Influenza and other respiratory virus surveillance systems in the Americas: 2017. https:// www.paho.org/hq/index.php?option=com docman\&view=download\&category_slug= scientific-technical-publications-5740\&alias= 42245-influenza-other-respiratory-virussurveillance-systems-americas-245\&Itemid= 270\&lang=en. Accessed 22 Feb 2021.

28. Martin-Loeches I, Schultz MJ, Vincent JL, et al. Increased incidence of co-infection in critically ill patients with influenza. Intensive Care Med. 2017;43:48-58.

29. Barros EN, Cintra O, Rossetto E, Freitas L, Colindres R. Patterns of influenza B circulation in Brazil and its relevance to seasonal vaccine composition. Braz J Infect Dis. 2016;20:81-90.

30. Ambrose CS, Levin MJ. The rationale for quadrivalent influenza vaccines. Hum Vaccin Immunother. 2012;8:81-8.

31. Gauci C, Melillo Fenech T, Gilles H, et al. Sentinel Surveillance: an option for surveillance of infectious intestinal disease. Euro Surveill. 2007;12: E13-4.

\section{Publisher's Note}

Springer Nature remains neutral with regard to jurisdictional claims in published maps and institutional affiliations. 